\title{
VULNERABILIDADE MENTAL NA AMBIÊNCIA ONCOLÓGICA: REVISÃO SISTEMÁTICA COM META-ANÁLISE E META-REGRESSÃO
}

\author{
João Ygor de Oliveira ${ }^{1}$, Modesto Leite Rolim Neto ${ }^{2}$
}

Introdução: Quais situações psíquicas poderiam ter rompido o ciclo de desamparo na ambiência oncológica, particularmente interligadas ao paciente em final de vida? Uma delas seria aquelas que apresentaram maior vulnerabilidade mental no processo de tratamento, desembocando em diferentes reações e sensações no processo de adoecimento. Objetivo: Analisar a influência da vulnerabilidade mental na prática de cuidados paliativos em pacientes oncológicos, utilizando os protocolos Preferred Reporting Items for Systematic Reviews and Meta-Analyses (PRISMA), bem como as recomendações do The National Institute for Health and Clinical Excellence (NICE), no avaliar intervenções únicas ou múltiplas a partir das quais as evidências foram obtidas. Método: Trata-se de uma revisão sistemática com meta-análise e metaregressão. A fonte de informações para os dados pesquisados foram a PubMed, Scopus, Web of Science e PsycINFO. A pesquisa foi realizada com os descritores MeSH: "Palliative Care", "Mental Health", "Mental Vulnerability", "Oncology". Foi utilizada a limitação de tempo entre 2012 a 2017 e o risco de viés dos estudos selecionados foram analisados segundo a escala de Downs e Black (1998). Para análise estatística foi utilizado o programa Stata 14.0 em que foi calculado a meta-análise pelo método de efeitos aleatório, metarregressão para analisar associação entre as variáveis analisadas e metabias para análise de viés dos resultados apresentados. Resultados: Entre os transtornos mentais mais comuns entre os pacientes oncológicos em cuidados paliativos estão os transtornos de adaptação, transtornos depressivos e transtornos ansiosos. Uma estimativa de efeito de 3,5\% é verificado nos pacientes com sensação de esgotamento, alterações do sono, conflitos nos relacionamentos e dúvidas existenciais. Quando verificado a associação entre transtorno mental e vulnerabilidade mental houve um resultado estatisticamente significativo $(\mathrm{p}=0,046)$ quando observado a persistência da ansiedade no tratamento. Analisando o resultado da metaregressão, pode-se notar que há um aumento de 56,9\% de risco para a inserção da vulnerabilidade mental quando são envolvidos questões pontuais relacionadas ao processo de morte e morrer. Conclusão: Pacientes oncológicos com algum grau de vulnerabilidade mental, a depender do estágio da doença e do tratamento, necessitam de suporte afetivo e emocional naquilo que circunda a prática de cuidados paliativos.

Palavras-chave: Cuidados Paliativos; Saúde Mental; Vulnerabilidade mental; Oncologia.

\footnotetext{
${ }^{1}$ Acadêmico de Enfermagem, Faculdade de Juazeiro do Norte - FJN;

2 Estácio/ Faculdade de Medicina de Juazeiro do Norte - FMJ;

Autor correspondente: ygorjoao57@gmail.com.
}

27 Id on Line Rev. Mult. Psic. V.12, N. 40. 2018 - ISSN 1981-1179 EDIÇÃO ESPECIAL: I CURSO DE ONCOLOGIA DO CARIRI / II JORNADA DE PESQUISA QUANTI-QUALITATIVA EM ONCOLOGIA. JUAZEIRO DO NORTE, 05 A 10 DE MARÇO DE 2018. Edição eletrônica em http://idonline.emnuvens.com.br/id 